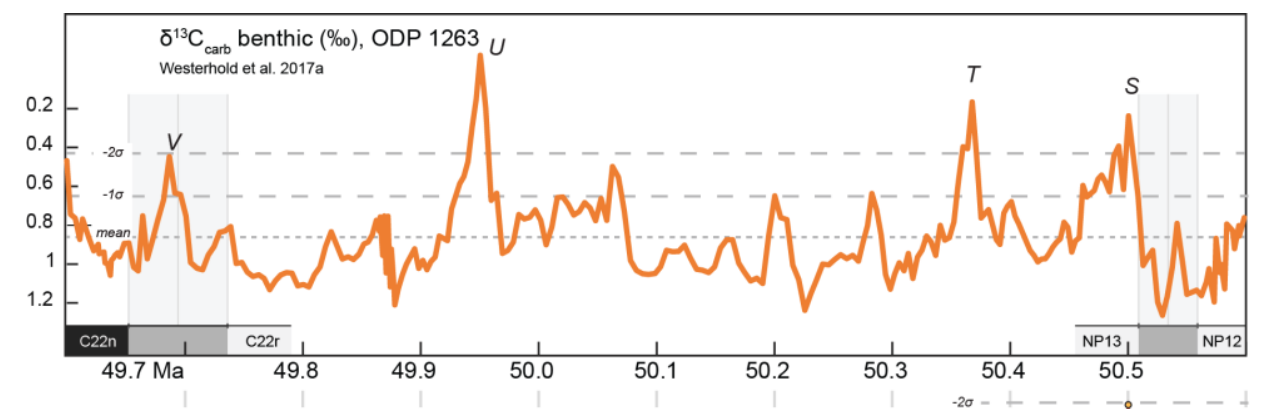

Sedimentation rate $(\mathrm{mm} / \mathrm{yr})$
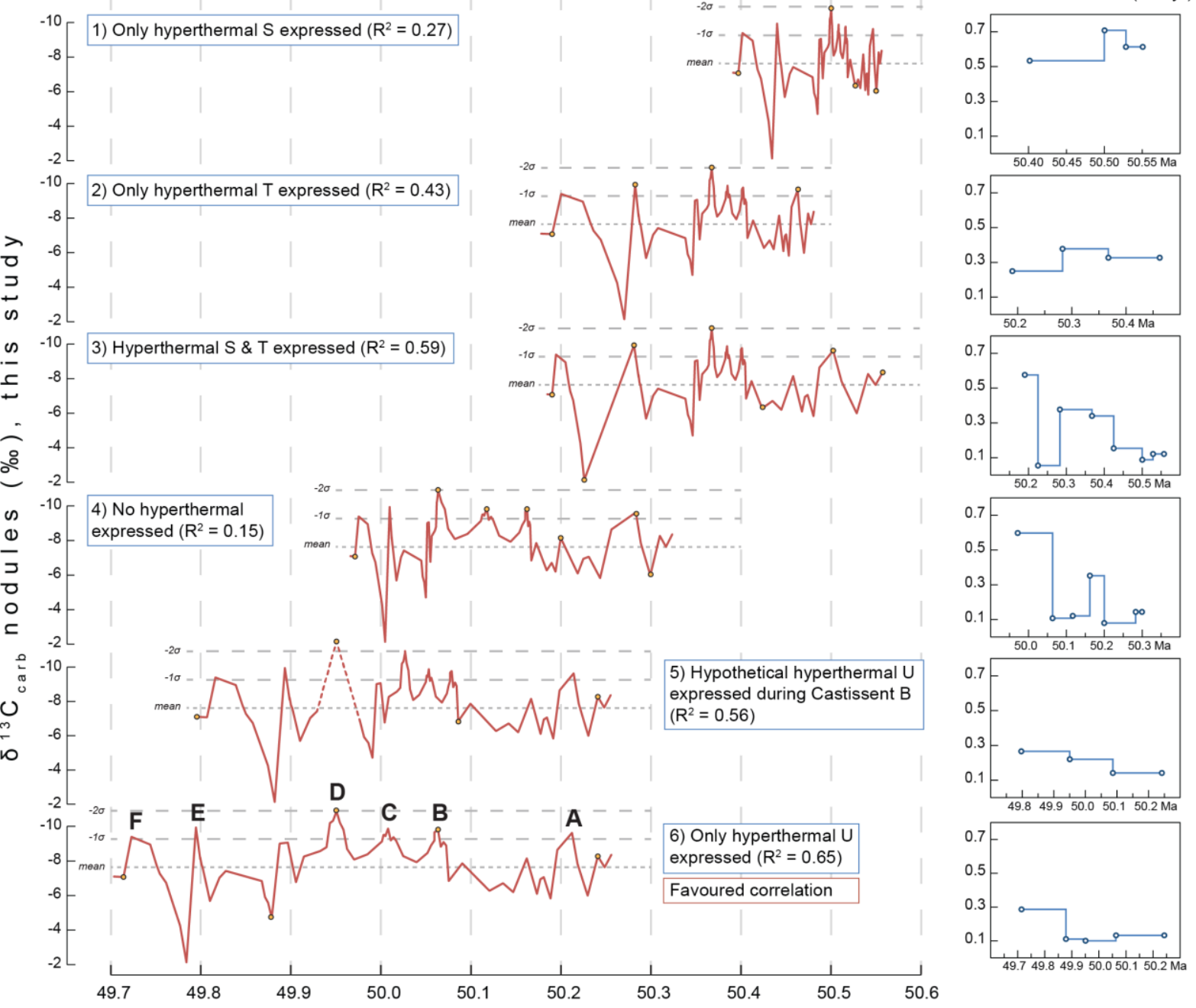

Figure S1: Correlation options 1 to 6 between $\delta^{13} \mathrm{C}$ benthic record from site 1263 and $\delta^{13} \mathrm{C}$ from carbonate nodules (this study) using the Analyseries software (Paillard et al., 1996). Tie-points used to correlate both curves are showed in yellow dots. Sedimentation rate in $\mathrm{mm} / \mathrm{yr}$ for each option is showed on the right. Option 6 is detailed in Figure 8. 


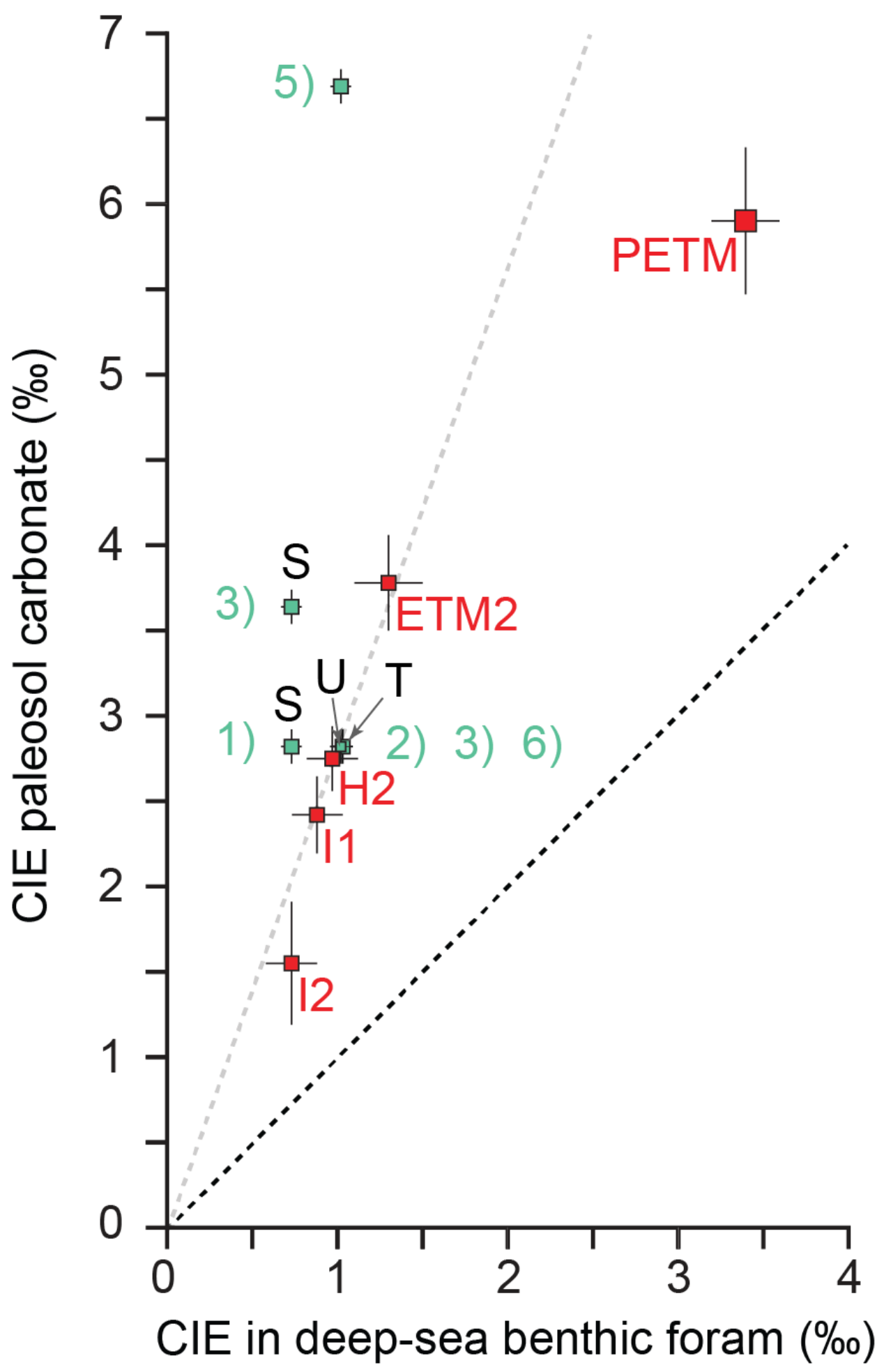

Figure S2: CIE amplitudes from I1, I2, H2, ETM2 and PETM in continental and marine environments after (Abels et al., 2016). Options 1 to 6 from Figure $S 1$ are plotted as comparison. 


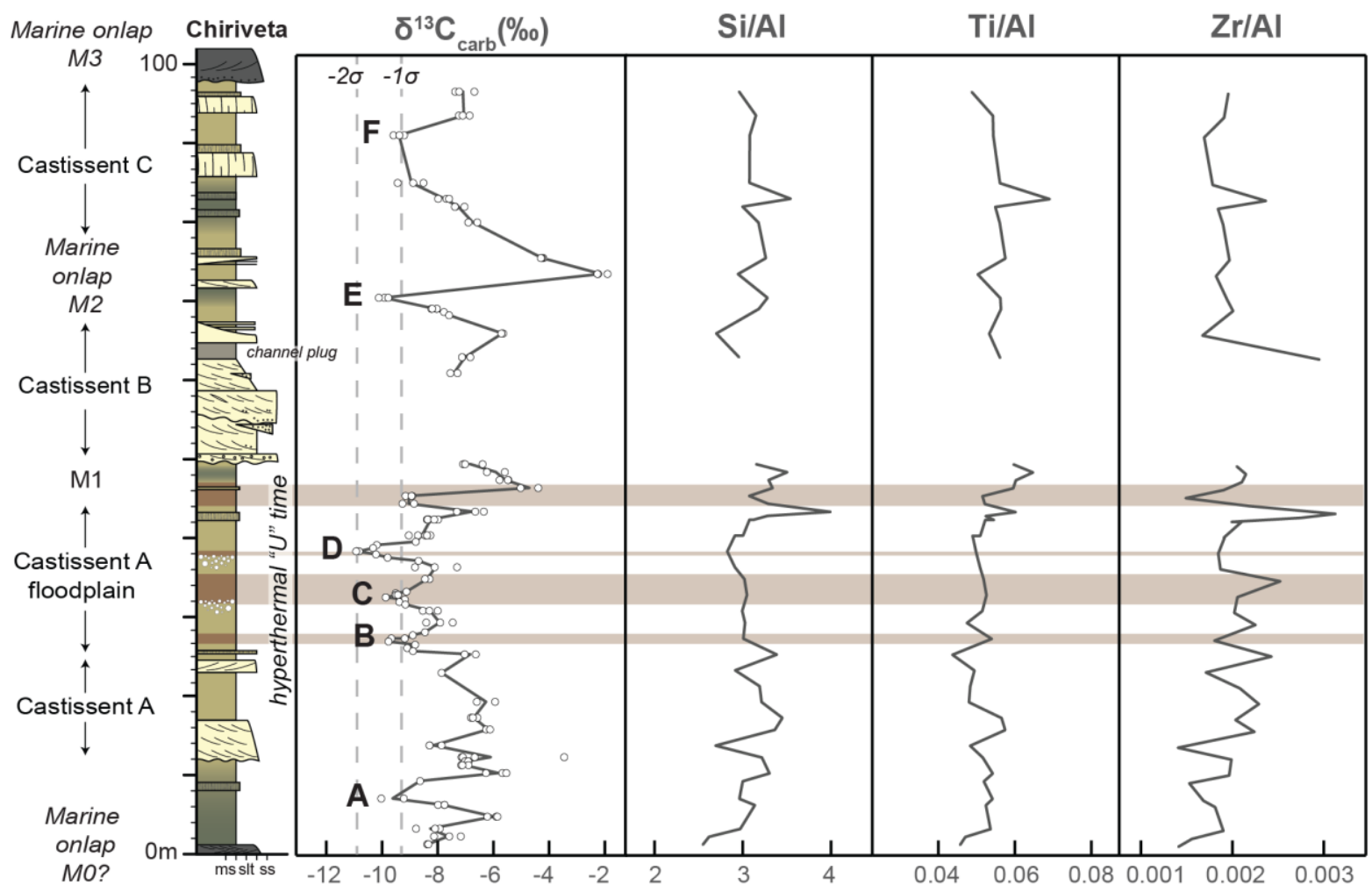

Figure S3: Chiriveta section showing $\delta^{13} \mathrm{C}$ and grain-size proxies $\mathrm{Si} / \mathrm{Al}$, Ti/Al and $\mathrm{Zr} / \mathrm{Al}$. Al is more concentrated in the fines and $\mathrm{Si}$, $\mathrm{Ti}$ and $\mathrm{Zr}$ in the coarse fraction of the sediment (Lupker et al., 2012). An increase of these ratios would suggest an increase in grain-size. Here, no direct correlation is observable between the grain size and the NCIEs. 


\begin{tabular}{|c|c|c|c|c|c|c|c|c|c|c|c|}
\hline samples & Phyllosilicates & Quartz & Feldspath-K & Plg-Na & Calcite & Indosés & Smectite & Illite-Smectite & Mica & Chlorite & Kaolinite \\
\hline MO4 & 21.57 & 15.74 & 0.00 & 0.00 & 53.96 & 8.71 & 31.04 & 2.41 & 24.11 & 20.30 & 22.11 \\
\hline MO5 & 13.71 & 19.58 & 0.00 & 0.00 & 62.52 & 4.17 & 26.73 & 5.85 & 20.78 & 33.83 & 12.78 \\
\hline MO7 & 32.57 & 39.76 & 3.07 & 1.03 & 20.56 & 2.99 & 26.96 & 2.92 & 32.95 & 29.87 & 7.27 \\
\hline
\end{tabular}

Table S1: Clay minerals results from sample MO2 to MO7 in the top of the Castissent Formation Member A, between 44 and 50m. From (Nicolaides, 2017). 


\begin{tabular}{|c|c|c|c|c|c|}
\hline sample & height (m) & Al (ppm) & Ti (ppm) & Fe (ppm) & $\mathrm{Zr}$ (ppm) \\
\hline $2 \mathrm{CH} 21$ & 96 & 35722.03886 & 1741.232797 & 13004.05365 & 69.80703089 \\
\hline $2 \mathrm{CH} 20$ & 93 & 53399.22227 & 2896.887595 & 26241.90692 & 102.0027324 \\
\hline $2 \mathrm{CH} 19$ & 90.5 & 62323.49447 & 3386.62809 & 28122.79949 & 105.4156279 \\
\hline $2 \mathrm{CH} 18$ & 84.5 & 70062.92314 & 3928.547221 & 32833.31428 & 125.1045792 \\
\hline 2CH17 & 82.5 & 49829.84454 & 3442.319098 & 30055.24786 & 117.9007775 \\
\hline 2 CH16 & 81.5 & 62653.58908 & 3440.134427 & 31770.77741 & 115.3843071 \\
\hline $2 \mathrm{CH} 15$ & 79.5 & 59648.45808 & 3344.117529 & 30092.96791 & 113.202646 \\
\hline S093 & 75 & 37316.28923 & 2145.734909 & 19819.63077 & 73.4467328 \\
\hline S092 & 73 & 36465.96172 & 1834.340824 & 16910.80025 & 66.38333503 \\
\hline S091 & 70 & 83411.30378 & 4687.47299 & 48621.43875 & 162.1496165 \\
\hline S090 & 68.6 & 66755.91805 & 3762.294958 & 35064.37338 & 133.9392266 \\
\hline S089 & 65.5 & 52205.89985 & 2782.934668 & 19424.12216 & 87.26031958 \\
\hline S088 & 62.5 & 66016.96245 & 3703.888199 & 40550.41391 & 194.6909276 \\
\hline MO2 & 49 & 57079.9901 & 3407.42471 & 26358.369 & 116.9071497 \\
\hline MO3 & 48 & 78503.73464 & 5080.617227 & 44616.41448 & 168.7810022 \\
\hline MO4 & 47 & 38632.68297 & 2326.88156 & 23423.75105 & 81.21215023 \\
\hline MO5 & 46 & 35666.23464 & 2124.793449 & 20252.2885 & 67.93857857 \\
\hline MO6 & 45 & 67280.75429 & 3467.53334 & 36062.46073 & 100.2098822 \\
\hline MO7 & 44 & 73928.46172 & 3857.491095 & 30310.15318 & 161.0371853 \\
\hline MO8 & 43 & 40822.67569 & 2456.380655 & 22990.56997 & 127.6102494 \\
\hline MO9 & 42.5 & 61266.27103 & 3209.862852 & 22214.42571 & 168.4785937 \\
\hline MO10 & 42 & 70736.94061 & 3856.054765 & 37479.31295 & 141.1430283 \\
\hline CH13 & 42 & 77156.68224 & 4030.381862 & 37161.98495 & 162.0632141 \\
\hline MO11 & 40 & 76820.89258 & 3912.132013 & 35009.47757 & 146.3703747 \\
\hline CH12 & 40 & 70740.43406 & 3462.850178 & 28819.91624 & 135.4944701 \\
\hline CH11 & 38 & 94884.28413 & 4727.0264 & 37525.7874 & 175.0883788 \\
\hline CH10 & 36 & 75584.06579 & 3847.34022 & 23751.18468 & 141.2618316 \\
\hline СH9 & 34.5 & 72862.99592 & 3776.151325 & 31488.65732 & 183.8798249 \\
\hline CH8 & 32.5 & 98856.93697 & 5195.789225 & 44653.47795 & 203.2555674 \\
\hline CH7 & 30.5 & 64223.7492 & 3307.17124 & 29947.58776 & 129.7271087 \\
\hline CH6 & 29 & 65052.67933 & 3088.124771 & 23421.83841 & 146.4675774 \\
\hline CH5 & 27 & 91510.81252 & 4934.521874 & 47587.70576 & 164.9144939 \\
\hline CH4 & 25 & 69228.18879 & 3026.31427 & 27584.51801 & 168.0033804 \\
\hline CH3 & 23 & 90131.50029 & 4454.521874 & 46650.11893 & 154.1357921 \\
\hline $\mathrm{CH} 2$ & 21 & 73852.82489 & 3569.186931 & 32013.51221 & 153.70378 \\
\hline CH1 & 19 & 69157.59199 & 3319.120546 & 31197.06916 & 158.4127118 \\
\hline $2 \mathrm{CH} 14$ & 17 & 61630.97263 & 3484.588259 & 25148.70109 & 125.4393886 \\
\hline $2 \mathrm{CH} 13$ & 15.5 & 60996.63857 & 3505.107269 & 31762.80329 & 136.7149043 \\
\hline $2 \mathrm{CH} 12$ & 13.5 & 67079.02576 & 3240.508597 & 30127.1862 & 94.20491403 \\
\hline 2 CH11 & 12 & 46807.48282 & 2417.816986 & 18984.95573 & 93.08168258 \\
\hline $2 \mathrm{CH} 10$ & 10 & 52672.2754 & 2856.175465 & 25014.74924 & 103.4931741 \\
\hline $2 \mathrm{CH} 9$ & 9 & 67219.21863 & 3487.11089 & 30697.52591 & 102.8235554 \\
\hline $2 \mathrm{CH} 8$ & 6.8 & 75173.34891 & 4071.347462 & 35723.89379 & 126.6166216 \\
\hline $2 \mathrm{CH} 7$ & 6 & 56945.40131 & 2984.008899 & 25258.15965 & 103.0827626 \\
\hline $2 \mathrm{CH} 6$ & 3 & 47496.96608 & 2547.15917 & 20364.47805 & 90.30600486 \\
\hline $2 \mathrm{CH} 5$ & 2 & 52020.0738 & 2448.148911 & 28009.1921 & 81.0825466 \\
\hline $2 \mathrm{CH} 4$ & 1 & 61612.41368 & 2815.692664 & 30977.08629 & 86.75270537 \\
\hline
\end{tabular}




\begin{tabular}{|c|c|c|c|c|c|c|c|}
\hline & & $\delta^{13} \mathrm{C}$ & $\delta^{18} O$ & & & $\delta^{13} \mathrm{C}$ & $\delta^{18} O$ \\
\hline sample & height (m) & \%о VPDB & \% VPDB & sample & height (m) & \%о VPDB & \%о VPDB \\
\hline 2CH21-P1 & 96 & -7.4 & -5.2 & MO5-G1 & 46 & -4.39 & -6.34 \\
\hline 2CH21-P2 & 96 & -7.2 & -5.0 & MO5-G2 & 46 & -5.02 & -6.20 \\
\hline 2CH21-G3 & 96 & -6.7 & -6.1 & MO6-P1 & 45 & -9.16 & -5.64 \\
\hline 2CH20-P1 & 93 & -7.2 & -6.3 & MO6-P2 & 45 & -8.92 & -5.65 \\
\hline $2 \mathrm{CH} 20 \mathrm{P} 2$ & 93 & -7.1 & -5.4 & MO6-P3 & 45 & -8.94 & -5.65 \\
\hline 2CH20-G3 & 93 & -6.9 & -5.5 & MO7-P1 & 44 & -8.85 & -5.19 \\
\hline 2CH19-P1 & 90.5 & -9.2 & -5.7 & MO7-P2 & 44 & -9.27 & -5.28 \\
\hline 2CH19-G2 & 90.5 & -9.6 & -5.7 & MO8-P1 & 43 & -7.31 & -5.89 \\
\hline 2CH19-G3 & 90.5 & -9.4 & -5.7 & MO8-P2 & 43 & -6.65 & -5.88 \\
\hline 2CH18-P1 & 84.5 & -8.5 & -6.1 & MO8-G3 & 43 & -6.35 & -5.99 \\
\hline 2CH18-P2 & 84.5 & -9.4 & -5.9 & MO10-P1 & 42 & -8.33 & -5.93 \\
\hline 2CH18-P3 & 84.5 & -8.9 & -6.0 & MO10-P2 & 42 & -8.32 & -6.06 \\
\hline 2CH17-P1 & 82.5 & -7.7 & -6.3 & MO10-P3 & 42 & -8.39 & -6.00 \\
\hline 2CH17-P2 & 82.5 & -8.0 & -6.2 & CH13-P3 & 42 & -7.99 & -5.83 \\
\hline 2CH17-G3 & 82.5 & -7.6 & -6.4 & $\mathrm{CH} 13-\mathrm{P} 2$ & 42 & -8.36 & -5.86 \\
\hline 2CH16-P1 & 81.5 & -7.4 & -6.5 & CH13-P1 & 42 & -8.13 & -5.39 \\
\hline 2CH16-P2 & 81.5 & -7.0 & -6.2 & MO11-P1 & 40 & -8.45 & -5.64 \\
\hline 2CH16-G3 & 81.5 & -7.4 & -6.4 & MO11-P2 & 40 & -8.27 & -5.95 \\
\hline 2CH15-P1 & 79.5 & -6.9 & -6.5 & CH12-P3 & 40 & -8.71 & -5.31 \\
\hline 2CH15-P2 & 79.5 & -6.6 & -6.6 & $\mathrm{CH} 12-\mathrm{P} 2$ & 40 & -8.38 & -5.84 \\
\hline 93-P1 & 75 & -4.2 & -6.1 & CH12-P1 & 40 & -9.04 & -5.16 \\
\hline 93-P2 & 75 & -4.3 & -6.1 & RB3-7 & 39.2 & -8.79 & -6.07 \\
\hline 93-P3 & 75 & -4.3 & -6.3 & RB3-6 & 38.8 & -10.19 & -5.94 \\
\hline 92-P1 & 73 & -1.9 & -6.4 & RB3-5 & 38.4 & -10.33 & -5.90 \\
\hline 92-P2 & 73 & -2.2 & -6.4 & RB3-4B & 38 & -10.82 & -5.89 \\
\hline 92-P3 & 73 & -2.3 & -6.4 & RB3-4A & 38 & -10.93 & -5.85 \\
\hline 91-P1 & 70 & -9.9 & -5.7 & RB3-3 & 37.6 & -10.22 & -6.01 \\
\hline 91-P2 & 70 & -10.1 & -6.0 & RB3-2 & 37.2 & -9.80 & -6.33 \\
\hline 91-G3 & 70 & -9.8 & -6.3 & RB3-1 & 36.8 & -8.69 & -6.31 \\
\hline 90-P1 & 68.6 & -8.0 & -6.1 & $\mathrm{CH} 10-\mathrm{G} 3$ & 36 & -7.31 & -6.24 \\
\hline 90-P2 & 68.6 & -8.0 & -6.1 & CH10-P2 & 36 & -8.11 & -5.95 \\
\hline 90-P3 & 68.6 & -8.2 & -6.0 & CH10-P1 & 36 & -8.82 & -5.93 \\
\hline RB4-3 & 68.6 & -8.21 & -5.50 & CH9-P2 & 34.5 & -8.29 & -6.14 \\
\hline RB4-2 & 68.2 & -7.78 & -5.96 & CH9-P1 & 34.5 & -8.46 & -6.36 \\
\hline RB4-1 & 67.8 & -7.59 & -6.26 & RB2-7 & 32.9 & -9.12 & -6.45 \\
\hline 89-P1 & 65.5 & -5.7 & -6.3 & RB2-6 & 32.7 & -9.51 & -6.14 \\
\hline 89-P2 & 65.5 & -5.7 & -6.4 & RB2-5B & 32.5 & -9.50 & -6.16 \\
\hline 88-P1 & 62.5 & -6.8 & -6.2 & RB2-5A & 32.5 & -9.43 & -5.98 \\
\hline $88-\mathrm{G} 2$ & 62.5 & -7.1 & -6.1 & RB2-4 & 32.2 & -9.87 & -5.75 \\
\hline 88-G3 & 62.5 & -7.1 & -6.2 & RB2-3 & 31.9 & -9.18 & -5.99 \\
\hline 87-P1 & 60.5 & -7.5 & -6.5 & RB2-2 & 31.6 & -9.38 & -5.80 \\
\hline 87-P2 & 60.5 & -7.3 & -6.4 & RB2-1 & 31.3 & -9.16 & -5.78 \\
\hline MO2-P1 & 49 & -7.09 & -5.56 & CH7-P3 & 30.5 & -8.53 & -5.92 \\
\hline MO2-P2 & 49 & -7.02 & -5.69 & CH7-P2 & 30.5 & -8.00 & -6.01 \\
\hline MO2-P3 & 49 & -6.39 & -5.82 & CH7-P1 & 30.5 & -8.30 & -5.98 \\
\hline MO3-P1 & 48 & -5.59 & -5.97 & CH6-P3 & 29 & -8.41 & -6.10 \\
\hline MO3-P2 & 48 & -6.23 & -5.75 & CH6-P2 & 29 & -7.46 & -6.16 \\
\hline MO4-P1 & 47 & -5.78 & -5.12 & CH6-G1 & 29 & -7.91 & -6.04 \\
\hline MO4-P2 & 47 & -5.46 & -5.13 & RB1-7 & 27.8 & -8.46 & -6.76 \\
\hline MO4-P3 & 47 & -5.48 & -5.20 & & & & \\
\hline
\end{tabular}




\begin{tabular}{|c|c|c|c|}
\hline & & $\delta^{13} \mathrm{C}$ & $\delta^{18} O$ \\
\hline sample & height (m) & \%о VPDB & \%о VPDB \\
\hline RB1-6 & 27.4 & -8.90 & -6.83 \\
\hline RB1-5B & 27 & -9.19 & -6.10 \\
\hline RB1-5A & 27 & -9.67 & -6.01 \\
\hline RB1-4 & 26.6 & -9.77 & -6.01 \\
\hline RB1-3 & 26.2 & -8.82 & -6.17 \\
\hline RB1-2 & 25.8 & -9.09 & -6.35 \\
\hline RB1-1 & 25.4 & -8.89 & -5.95 \\
\hline CH4-P2 & 25 & -6.64 & -6.49 \\
\hline CH4-P1 & 25 & -7.04 & -6.16 \\
\hline CH3-P1 & 23 & -7.86 & -5.31 \\
\hline CH1-P2 & 19 & -5.94 & -6.06 \\
\hline CH1-P1 & 19 & -6.60 & -6.03 \\
\hline 2CH14-P1 & 17 & -6.6 & -5.7 \\
\hline 2CH14-P2 & 17 & -6.8 & -5.8 \\
\hline 2CH14-P3 & 17 & -6.7 & -5.6 \\
\hline 2CH13-P1 & 15.5 & -6.3 & -6.5 \\
\hline 2CH13-P2 & 15.5 & -6.1 & -6.7 \\
\hline 2CH12-P1 & 13.5 & -8.3 & -5.3 \\
\hline 2CH12-P2 & 13.5 & -8.3 & -5.4 \\
\hline 2CH12-G3 & 13.5 & -7.9 & -5.4 \\
\hline 2CH3-P1 & 12 & -3.5 & -7.0 \\
\hline 2CH11-P1 & 12 & -7.1 & -5.9 \\
\hline 2CH11-P2 & 12 & -6.7 & -5.9 \\
\hline 2CH11-G3 & 12 & -7.1 & -5.8 \\
\hline 2CH1-P1 & 11.5 & -6.9 & -5.5 \\
\hline 2CH1-P2 & 11.5 & -7.0 & -5.5 \\
\hline 2CH1-P3 & 11.5 & -6.9 & -5.5 \\
\hline 2CH2-P1 & 11 & -7.2 & -5.5 \\
\hline 2CH2-P2 & 11 & -6.9 & -5.5 \\
\hline 2CH2-P3 & 11 & -7.1 & -5.7 \\
\hline 2CH10-P1 & 10 & -5.7 & -6.6 \\
\hline 2CH10-P2 & 10 & -5.5 & -6.6 \\
\hline 2CH10-G3 & 10 & -6.3 & -6.6 \\
\hline 2CH9-P1 & 9 & -8.6 & -5.5 \\
\hline 2CH8-P1 & 6.8 & -10.0 & -5.1 \\
\hline 2CH8-P2 & 6.8 & -9.2 & -5.3 \\
\hline 2CH7-P1 & 6 & -8.0 & -5.9 \\
\hline 2CH7-P2 & 6 & -7.8 & -5.8 \\
\hline 2CH7-G3 & 6 & -7.8 & -5.7 \\
\hline 2CH6BIS-P1 & 4.5 & -5.9 & -6.5 \\
\hline 2CH6BIS-P2 & 4.5 & -6.2 & -6.4 \\
\hline 2CH6BIS-P3 & 4.5 & -5.9 & -6.6 \\
\hline 2CH6-P1 & 3 & -7.9 & -6.3 \\
\hline 2CH6-P2 & 3 & -8.8 & -6.2 \\
\hline 2CH6-G3 & 3 & -8.1 & -6.3 \\
\hline 2CH5-P1 & 2 & -7.6 & -6.3 \\
\hline 2CH5-P2 & 2 & -8.1 & -6.2 \\
\hline 2CH5-P3 & 2 & -7.2 & -6.3 \\
\hline 2CH4-P1 & 1 & -8.4 & -6.1 \\
\hline 2CH4-P2 & 1 & -8.3 & -6.3 \\
\hline
\end{tabular}

Table S2: Al, Ti, Fe, $\mathrm{Zr}, \delta^{13} \mathrm{C}$ and $\delta^{18} \mathrm{O}$ values of the Chiriveta section 


\section{References Supplement}

Abels, H. A., Lauretano, V., van Yperen, A. E., Hopman, T., Zachos, J. C., Lourens, L. J., Gingerich, P. D. and Bowen, G. J.: Environmental impact and magnitude of paleosol carbonate carbon isotope excursions marking five early Eocene hyperthermals in the Bighorn Basin, Wyoming, Clim. Past, 12(5), 1151-1163, doi:10.5194/cp-12-1151-2016, 2016

Lupker, M., France-Lanord, C., Galy, V., Lavé, J., Gaillardet, J., Gajurel, A. P., Guilmette, C., Rahman, M., Singh, S. K. and Sinha, R.: Predominant floodplain over mountain weathering of Himalayan sediments (Ganga basin), Geochim. Cosmochim. Acta, 84, 410-432, doi:10.1016/j.gca.2012.02.001, 2012.

Nicolaides, E.: Analyses des sédiments marins et continentaux éocènes dans les Pyrénées Espagnoles, Université de Lausanne., 2017.

Paillard, D., Labeyrie, L. and Yiou, P.: Macintosh Program performs time-series analysis, Eos, Trans. Am. Geophys. Union, doi:10.1029/96EO00259, 1996. 